\title{
1 Sirtuin-1 Sensitive Lysine-136 Acetylation Drives Phase Separation and Pathological \\ 2 Aggregation of TDP-43
}

3 Jorge Garcia Morato ${ }^{1,2}$, Friederike Hans ${ }^{1,2}$, Felix von Zweydorf ${ }^{2}$, Regina Feederle ${ }^{3,4}$, Simon J. Elsässer ${ }^{5}$,

4 Angelos A. Skodras ${ }^{6}$, Christian Johannes Gloeckner ${ }^{2,7}$, Emanuele Buratti ${ }^{8}$, Manuela Neumann ${ }^{2,9}$, Philipp

5 J. Kahle $1,2, *$

$6{ }^{1}$ Laboratory of Functional Neurogenetics, Department of Neurodegeneration, Hertie Institute for Clinical

7 Brain Research, University of Tübingen, 72076 Tübingen, Germany

$8{ }^{2}$ German Center for Neurodegenerative Diseases (DZNE), 72076 Tübingen, Germany

93 Institute for Diabetes and Obesity, Monoclonal Antibody Core Facility and Research Group, Helmholtz

10 Zentrum München, 85764 Neuherberg, Germany

$11{ }^{4}$ German Center for Neurodegenerative Diseases (DZNE), 81377 München, Germany

$12{ }^{5}$ Science for Life Laboratory, Department of Medical Biochemistry and Biophysics, Karolinska Institutet,

1317165 Stockholm, Sweden

$14{ }^{6}$ Molecular Imaging Unit, Department of Cellular Neurology, Hertie Institute for Clinical Brain Research,

15 University of Tübingen, 72076 Tübingen, Germany

$16{ }^{7}$ Core Facility for Medical Bioanalytics (CFMB), Institute for Ophthalmic Research, Center for

17 Ophthalmology, University of Tübingen, 72076 Tübingen, Germany

$18{ }^{8}$ International Centre for Genetic Engineering and Biotechnology (ICGEB), 34149 Trieste, Italy

$19{ }^{9}$ Department of Neuropathology, University Hospital, 72076 Tübingen, Germany

21 * Corresponding author. Tel: +49 7071 29-81970; E-mail: philipp.kahle@uni-tuebingen.de 


\section{Abstract}

25 The trans-activation response DNA-binding protein TDP-43 regulates RNA processing and forms neuropathological aggregates in patients with amyotrophic lateral sclerosis and frontotemporal lobar degeneration. Investigating TDP-43 post-translational modifications, we discovered that K84 acetylation reduced nuclear import whereas K136 acetylation impaired RNA binding and splicing capabilities of TDP-

43. Such failure of RNA interaction triggered TDP-43 phase separation mediated by the C-terminal low complexity domain, leading to the formation of insoluble aggregates with pathologically phosphorylated and ubiquitinated TDP-43. Confirming the results from site-directed mutagenesis, we succeeded to introduce authentic acetyl-lysine at the identified sites via amber suppression. [AcK84]TDP-43 showed cytoplasmic mislocalization and the aggregation propensity of [acK136]TDP-43 was confirmed. With newly developed antibodies, we found that the nuclear sirtuin SIRT1 can potently deacetylate [acK136]TDP-43. Moreover, SIRT1 reduced the aggregation propensity of [acK136]TDP-43. Thus, distinct lysine acetylations modulate nuclear import, RNA binding and phase separation of TDP-43, suggesting novel regulatory mechanisms for TDP-43 pathogenesis.

\section{Introduction}

The trans-activation response DNA-binding protein of $43 \mathrm{kDa}$ (TDP-43) regulates various RNA processing steps $^{1-3}$ and is found in the neuropathological lesions of patients with amyotrophic lateral sclerosis (ALS) and frontotemporal lobar degeneration (FTLD) ${ }^{4,5}$. The TDP-43 polypeptide contains two RNA recognition motifs (RRM1 and RRM2), a nuclear localisation signal (NLS) and a C-terminal glycine-rich low complexity domain (see Fig. 1a). The NLS allows active nuclear import ${ }^{6}$, but TDP-43 can also shuttle between the nucleus and cytoplasm ${ }^{7,8}$. Through its RRMs TDP-43 binds to UG-rich regions of RNA ${ }^{9,10}$ and it is involved in RNA transport, stability, and splicing, for example exon 9 of the cystic fibrosis transmembrane conductance regulator (CFTR) mRNA ${ }^{11}$. The C-terminal low complexity domain of TDP-43 promotes 
modifications (PTMs) can modulate TDP-43 functions in health and disease. Putative pathological PTMs of TDP-43 include C-terminal fragmentations and phosphorylations ${ }^{13}$, including the widely used marker pS409/41014,15. In addition lysine modifications have been reported, including ubiquitinations at various residues $^{16}$ and sumoylation and acetylation of the RRM1 ${ }^{17,18}$. The putative acetyl-mimic [K145Q]TDP-43 was distributed in a stippled manner in transfected cells, eventually recapitulating pathological phosphorylation and recruitment of ALS-related factors into TDP-43 aggregates ${ }^{19}$. In addition, [K145Q]TDP-43 had reduced CFTR splicing activity. Thus, lysine modifications may be important for physiology and pathological aggregation of TDP-43. However, the genesis of TDP-43 aggregates remains elusive.

New research on LLPS is unveiling the molecular processes regulating the arrangement of membraneless organelles. Heteronuclear ribonucleoprotein (hnRNP) LLPS is amply reported but whether and how this process is regulated in cells is poorly understood ${ }^{20}$. In the case of TDP-43, RNA binding prevents its phase separation in vitro and reduces droplet formation in vivo' ${ }^{21}$ Wild-type (wt)TDP-43 is capable of going into liquid droplets but pathological mutants seem to form more resilient droplets. While the C-terminal glycine-rich domain of TDP-43 seems to play a crucial role in the formation of phase-separated droplets, the $\mathrm{N}$-terminal domain may also contribute to the aggregation process ${ }^{22-24}$. Thus, the regulation of TDP-43 RNA binding could be pivotal in the pathophysiology of TDP-43. The molecular pathways regulating the disengagement from RNA are only beginning to be described.

In the present study we discovered that acetylation of K84 within the NLS reduced nuclear import whereas acetylation of K136 in the RNA recognition domain impaired TDP-43 RNA binding and splicing capabilities. Such failure of RNA interaction triggered TDP-43 phase separation mediated by the Cterminal low complexity domain, leading to the formation of insoluble aggregates with pathologically phosphorylated and ubiquitinated TDP-43. To confirm the results from site-directed mutagenesis, we expanded the genetic code via amber suppression to introduce authentic acetyl-lysine at the identified

75 sites. Indeed, [acK84]TDP-43 showed cytoplasmic mislocalization, and the increased aggregation 
nuclear sirtuin SIRT1 can potently deacetylate [acK136]TDP-43. Moreover, SIRT1 reduced the aggregation propensity of [acK136]TDP-43. Thus, distinct lysine acetylations regulate nuclear import, RNA binding and phase separation of TDP-43, suggesting novel mechanisms for TDP-43 pathogenesis.

\section{Results}

\section{Detection of TDP-43 acetylation}

83 After investigating the sites of TDP-43 lysine ubiquitinations ${ }^{16}$, we became interested in how other PTMs

84 could affect TDP-43 functionality and aggregation. Recently acetylation has been linked with TDP-43

85 pathology ${ }^{18,19}$, and lysine acetylation has the potential to disrupt ubiquitination by competing for the same

86 residue. To explore this hypothesis more in depth, we re-examined the mass spectrometry data ${ }^{16}$

87 specifically for acetylated lysines. We found in transfected HEK293E cells that 6xHis-tagged wtTDP-43

88 was acetylated at lysine residues K79 and K84 around the NLS and K121 and K136 in RRM1 (Fig. 1a).

89 We could not detect the previously published acetylations at K145 and K192 ${ }^{18}$, perhaps due to slight 90 differences in experimental conditions (see discussion).

\section{Site-directed mutagenesis of TDP-43 acetyl-lysine sites}

93 Each of the identified acetylated lysine residues was mutagenized to either arginine or glutamine to

94 simulate the lack or the presence of acetylation, respectively. We first looked at the subcellular distribution

95 of these N-terminally 6xHis-tagged TDP-43 mutants in transiently transfected HEK293E cells.

96 Immunofluorescence staining of the $6 \mathrm{xH}$ is tag did not show visible deviation from the wtTDP-43 nuclear

97 staining pattern for both acetyl-dead and acetyl-mimic mutations at residues $\mathrm{K} 79$ and $\mathrm{K} 121$

98 (Supplementary Fig. 1). Likewise, the conservative [K84R]TDP-43 mutant remained mainly nuclear, but a

99 substantial portion of acetyl-mimic [K84Q]TDP-43 was retained in the cytoplasm (Fig. 1b). Quantification

100 showed a significant increase in the percentage of cells with cytoplasmically mislocalized [K84Q]TDP-43

101 (Fig. 1c). In addition, we performed biochemical nucleo-cytoplasmic fractionation assays. There was a 
significant increase of [K84Q]TDP-43 in the cytoplasmic fraction when compared to wtTDP-43 (Fig. 1d-e).

103 Nevertheless, we could still observe a sizable amount of [K84Q]TDP-43 in the nuclear fraction, as for the

$104[\Delta$ NLS]TDP-43 triple mutant. Thus, alterations at the NLS reduced but did not abolish TDP-43 nuclear

105 import, accounting for the residual splicing activity in the nucleus (see Fig. 3e,f). By contrast, the acetyl-

106 mimic K79Q substitution did not show evident changes in nucleocytoplasmic distribution of TDP-43, likely

107 because K79 is localized just outside the NLS, whereas K84 is at the core of the NLS $6,16$.

The RRM1 mutants $\mathrm{K} 136 \mathrm{R}$ and $\mathrm{K} 136 \mathrm{Q}$ were predominantly localized in the nucleus, but a significant

110 number of cells displayed a nuclear droplet-like pattern (Fig. 1b,c). This droplet-like nuclear distribution

111 was reminiscent of the RNA-binding deficient F147L/F149L mutant TDP-438,25. For comparison, we

112 generated the previously described ${ }^{18,19}$ acetyl-mimic $\mathrm{K} 145 \mathrm{Q}$ mutant in RRM1, which showed the expected

113 stippled distribution (Fig. 1b). As K136 is in direct contact with bound nucleic acids ${ }^{26,27}$, we assume that

114 modifications at K136 disrupt nucleic acid binding and therefore disengage TDP-43 from hnRNP complex

115 localizations. Such dissociated K136-modified TDP-43 may be free to phase separate and self-aggregate

116 into inclusions.

K136 mutant TDP-43 is pathologically ubiquitinated, phosphorylated, and insoluble

We examined if the K136 mutant TDP-43 inclusions in cell culture also showed pathological features described for human patients ${ }^{4,5,14,28}$. 6xHis-tagged TDP-43 variants were transiently transfected into HEK293E cells with stable knockdown of endogenous TDP-43 (sh $\left.{ }^{\text {TDP-43 }}\right)^{29}$ to minimize interference from the endogenous wtTDP-43. To assess protein solubility, RIPA-urea solubility fractionation assays were performed. While most of the 6xHis-tagged wtTDP-43 was RIPA-soluble, a larger portion of both K136R

124 and K136Q TDP-43 mutants shifted into the RIPA-insoluble fraction (Fig. 2a). Although 125 [F147L/F149L]TDP-43 formed similar intranuclear patches, this designed RNA-binding deficient mutant 126 was as soluble as endogenous TDP-43 (Fig. 2a). It should be noted that the Flag-tagged 
127 [F147L/F149L]TDP-43 was expressed at levels comparable to endogenous TDP-43 in non-silenced cells,

128 whereas the 6xHis-tagged K136 mutants were expressed at higher levels.

130 To check for pathological PTMs, 6xHis-tagged TDP-43 variants were transiently transfected into sh ${ }^{\text {TDP-43 }}$

131 HEK293E cells and purified using nickel beads. Both K136R and K136Q TDP-43 were ubiquitinated more

132 strongly than wtTDP-43 even after proteasomal inhibition (Fig. 2b). In addition, K136R and K136Q mutant

133 TDP-43 showed phosphorylation at the extreme C-terminal serine residues S409/410 detected with a

134 phospho-specific antibody ${ }^{15}$ (Fig 2b). This pathological phosphorylation was also visible at the nuclear

135 inclusions of K136 mutant TDP-43 by immunostaining (Fig. 2c). Thus, the nuclear inclusions formed by

136 K136 mutant TDP-43 recapitulate PTMs found in patients.

K136 mutant TDP-43 fails to bind RNA and lacks splicing activity

Because K136 in the RRM1 is important for interaction with nucleic acids ${ }^{26,27}$, we hypothesised that 140 alterations at position K136 could interfere with RNA binding of TDP-43 and alter its splicing capabilities.

141 First, we performed a RNA-protein pulldown assay of TDP-43 with its preferred binding sequence poly$142(\mathrm{UG})_{12}{ }^{10}$. While wtTDP-43 strongly bound to synthetic poly-(UG) 12 but not the negative control poly-(UC) $)_{12}$ 143 RNA, the acetyl-mimic [K136Q]TDP-43 showed a reduction in RNA binding (Fig. 3a). Additionally, we 144 measured the RNA-binding affinity using a 2-filter trap assay using an excess of synthetic poly-(UG) 12 as 145 target. The first nitrocellulose membrane will capture RNA only when bound to protein and the second 146 nylon membrane captures the unbound flow-through RNA. This assay clearly showed the concentration147 dependent formation of [wt]TDP-43 protein complex with poly-(UG) ${ }_{12}$ RNA retained on the nitrocellulose 148 membrane (Fig. 3b). In contrast, the acetyl-mimic [K136Q]TDP-43 had significantly reduced poly-(UG) 12 149 RNA binding capacity (Fig. 2b-d). 
151 To assess the splicing activity of TDP-43 in cells, we used the established CFTR minigene reporter

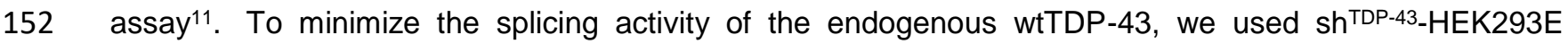

153 cells ${ }^{29}$. Mutant constructs of TDP-43 were co-transfected together with a plasmid containing exons 9-11 of

154 CFTR in a minigene. In parental, non-silenced cells exon 9 was skipped, which was severely blunted in 155 sh $^{\text {TDP-43 }}$ cells (Fig. 3e). Ex9 splicing could be rescued by re-transfecting [wt]TDP-43 (Fig. 3e). The nuclear

156 [K84R]TDP-43 rescued CFTR splicing to wild-type levels. [K84Q]TDP-43 rescued CFTR splicing activity to

157 a lesser extent than wild-type or [K84R]TDP-43 (Fig. 2h). The reduced splice activity of the nuclear import 158 impaired [K84Q]TDP-43 was similar to that of [ $\triangle$ NLS]TDP-43, suggesting that the residual nuclear TDP-43

159 (Fig. 1d) was sufficient to promote ex9 skipping. Indeed, in our experience very strong reduction of TDP-

16043 activity is necessary to cause loss of TDP-43 splice activity in cells. In contrast, both K136R and

$161 \mathrm{~K} 136 \mathrm{Q}$ mutants showed significantly reduced CFTR exon 9 splicing (Fig. 3e,f), linking the formation of 162 nuclear inclusions with TDP-43 loss of function. The previously described ${ }^{18,19} \mathrm{~K} 145 \mathrm{Q}$ mutant TDP-43 also 163 showed some reduction of CFTR splice activity, although the effect was less pronounced than for the 164 K136 mutants expressed at comparably high protein levels (Fig. 3e). Together, both CFTR splicing assay 165 and RNA-protein pulldown show that alterations at position 136 severely perturb the RNA-binding and 166 splicing capabilities of TDP-43. It was puzzling that both the acetyl-mimic K136Q and the acetyl-dead K136R substitutions showed the same aggregation-promoting effects. The K136 residue may be in a structurally restrained position not tolerating even conservative amino acid substitutions. Indeed, the NMR structure of the TDP-43 RRMs

171 complexed with RNA ${ }^{26}$ revealed that the side chain of $\mathrm{K} 136$ is in close apposition to the nucleic acid and 172 the key RNA-binding residues F147/F149 (Supplementary Fig. 2a). Moreover, K136 is within $\approx 3 \AA$ distance 173 to Q134, L139 and T199, evidently forming a tight RNA-binding configuration that appears not to 174 accommodate the larger guanidinium group of the otherwise conservative K136R substitution. 175 Supplementary Fig. 2b highlights the K136 residue within the RRM1 bound to RNA. Modelling the acetyl176 dead K136R mutation, which surprisingly caused similar aggregation propensities as the acetyl-mimicking 177 K136Q substitution, revealed clashes with the protein backbone. Thus, classical site-directed mutagenesis 
178 might introduce local perturbations to the RRM1 structure, which may not entirely reflect the effects of

179 authentic lysine acetylation. Indeed, the model for [acK136]TDP-43 showed hardly any clashes with the

180 protein backbone, but a collision with the bound RNA structure. In order to distinguish mutagenesis

181 artefacts from authentic lysine acetylation effects, we needed to introduce acK at position 136 of TDP-43.

Introduction of acetyl-Iysine (acK) through amber suppression

As K136 turned out to be a very delicate residue for site-directed mutagenesis, it was important to properly

validate the pro-aggregative effects for [acK136]TDP-43. We succeeded to incorporate authentic acK at the apparently regulatory sites in TDP-43 with amber suppression methodology. TDP-43 mutants were generated with a C-terminal 6xHis tag and an amber stop codon (TAG) substituting the codon at position

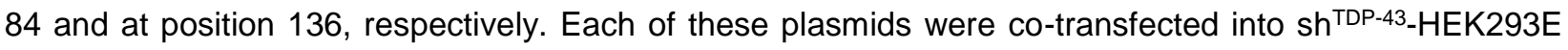
cells, together with a plasmid containing a N-terminally Flag-tagged chimeric acetylated lysine RNA synthetase (AcRS) and four copies of $\operatorname{tRNA}_{\mathrm{TAG}}{ }^{30}$. Together the designed AcRS and the $t R N A_{T A G}$ will incorporate acK added to the media at the introduced amber stop codon, directly incorporating an acetylated lysine site-specifically during the protein translation.

In absence of acK, only truncated forms of TDP-43 were visible due to the introduction of the amber stop codons (Fig. 4a). After adding $5 \mathrm{mM}$ acK in the media for $24 \mathrm{~h}$, the constructs were translated beyond the amber sites such that full-length TDP-43 including the C-terminal 6xHis tag became detectable, confirming successful amber suppression (Fig. 4a). There was a reduction in the amount of truncated TDP-43 but it did not completely disappear, pointing out the slight inefficiency of the system when compared to endogenous tRNAs. The expression of amber-suppressed full-length mutant TDP-43 was not as high as

200 that from a wtTDP-43 cDNA (Fig. 4a), but still robust. Importantly, [acK84]TDP-43 showed a similar reduction of nuclear import (Fig. 4b) as the K84Q mutant (Fig. 1b), and [acK136]TDP-43 showed the same 
capabilities of TDP-43 were compromised after introducing an acetylated lysine at position 136 in a similar

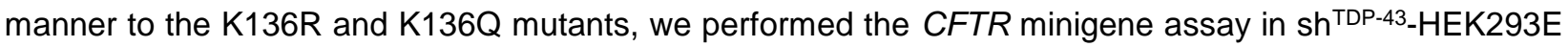
cells. Indeed, [acK136]TDP-43 could only partially rescue CFTR exon 9 splicing (Fig. 4c). In addition, we also introduced acetylated lysine at K79 and K121 (Supplementary Fig. 3). As expected from the point mutations (Supplementary Fig. 1), [acK79]TDP-43 and [acK121]TDP-43 did not show any different distribution than wtTDP-43. Taken together, the acetyl-mimic lysine-to-glutamine substitutions did reflect authentic lysine acetylation properties, as confirmed by amber suppression introduction of acK directly.

We were then interested if [acK136]TDP-43 could form phase-separated droplets or even aggregates in the cytoplasm, the most common site where human neuropathological TDP-43 is found ${ }^{31}$. To study this, we disrupted the NLS ${ }^{6}$ in the construct $\Delta$ NLS-K136 ${ }_{\text {TAG. }}$ [AcK136)TDP-43 $\Delta$ NLS was uniformly distributed throughout the cytoplasm in most cells. However, there was a fraction of cells developing multiple cytoplasmic aggregates (Fig. 4e,f). Thus, when nuclear import is impaired, K136 acetylation may also promote aggregation of cytosolically mislocalized TDP-43. Next, we deleted the C-terminus in the construct $\Delta \mathrm{C}-\mathrm{K} 136_{\mathrm{TAG}}$ because the $\mathrm{C}$-terminal low complexity domain mediates LLPS and aggregation of

218 TDP-43 ${ }^{32}$. [acK136)TDP-43 $\Delta \mathrm{C}$ showed a homogeneous nuclear distribution (Fig. 4e,f), confirming that the 219 C-terminal part of TDP-43 plays a crucial role in phase separation and aggregation.

As we confirmed that [K136Q]TDP-43 showed the same properties as amber suppressed [acK136]TDP[K136Q]TDP-43 construct. First we measured protein motility in the droplet-like inclusions by fluorescence recovery after photobleaching assay (FRAP). HEK293E cells were transfected with either wtTDP-43- 
228 minute after photobleaching (Fig. 5b), consistent with the motility of TDP-43 in the nucleus ${ }^{21}$. By contrast,

229 two types of less mobile pools of [K136Q]TDP-43-EGFP were detected (Fig. 5b). Large [K136Q]TDP-43-

230 EGFP aggregates $(\varnothing \geq 1 \mu \mathrm{m})$ did not recover for up to 3 min recording (Fig. 5b), likely constituting solid

231 aggregates. The smaller punctae $(\varnothing \leq 0.5 \mu \mathrm{m})$ showed a significantly slower recovery than the wtTDP-43

232 with an immobile fraction not recovering at all (Fig. 5b-c), indicative of LLPS ${ }^{21}$.

234 To investigate the kinetics of LLPS and protein aggregation, we performed long-term live imaging of 235 [K136Q]TDP-43-EGFP. Transfected HEK293E cells were imaged for several hours and the distribution of 236 TDP-43 was monitored. [K136Q]TDP-43 was recorded going into phase separation, forming droplets and 237 these droplets were seen fusing with one another (Fig. 5d and Supplementary Videos 1-3). To examine 238 the [K136Q]TDP-43 aggregate-formation kinetics in detail we quantified the size of aggregates in 239 HEK293E cells fixed 24, 48 and 72 hours after transfection. The quantification showed that after 48 hours 240 there was an increase in the percentage of TDP-43 aggregates larger than $10 \mu \mathrm{m}$, and the size difference 241 became significant after 72 hours (Fig $5 \mathrm{e}$ ). Together, these results suggest that the aggregation of TDP24243 is a dynamic process from a diffuse distribution to phase separation, eventually towards larger solid 243 aggregates.

\section{Generation of site-specific antibodies against lysine acetylated TDP-43}

246 To study the acetylated forms of TDP-43 in greater detail, we developed rat monoclonal antibodies against 247 acK84 and acK136 TDP-43. Two clones were selected that recognized with high sensitivity their 248 corresponding amber suppressed TDP-43 targets (Fig. 6a,b) in Western blots, with a very faint band 249 visible in the wtTDP43 lane. The antibodies did not recognize K84R or K136R TDP-43 mutants, 250 respectively. Both antibodies were also capable of detecting the corresponding lysine acetylated forms of 251 TDP-43 by immunofluorescence staining (Fig. 6c,d). 
254 The newly generated antibodies allowed us to check for enzymes that could deacetylate [acK136]TDP-43.

255 In humans, there are 18 protein lysine deacetylases ${ }^{33}$. First we tested the histone deacetylases (HDACs)

$2561-8$, representing class I and class II deacetylases. Each of these HDACs was cotransfected into amber

257 suppressed [acK136]TDP-43 cells. None of the HDAC1-8 effectively reduced TDP-43 K136 acetylation,

258 (Supplementary Fig. 4a). However, the results for Myc-tagged HDAC1 and HDAC6 were somewhat

259 inconclusive as their expression interfered with the amber suppression efficiency for [acK136]TDP-43

260 expression (Supplementary Fig. 4b). Next, we tested all class III deacetylases (sirtuins), also because of

261 their involvement in neurodegenerative diseases including ALS ${ }^{34}$. Flag-tagged SIRT1-7 were co-

262 transfected with K136TAG and the deacetylation effects on amber-suppressed [acK136]TDP-43 were

263 probed with the acetylation-specific antibody. Among the 7 sirtuins, only SIRT1 and SIRT2 strongly

264 reduced [acK136]TDP-43 immunoreactivity (Supplementary Fig. 4c). To confirm that the reduction in K136

265 acetylation was truly caused by the deacetylation activity of these sirtuins, we repeated the experiment in

266 the presence of Ex527, a selective Sirt1 and to a lesser extent Sirt2 inhibitor. Indeed, treatment with

267 Ex527 dose-dependently reduced the deacetylation of [acK136]TDP-43 by SIRT1 and SIRT2 (Fig 7a).

268 [AcK136]TDP-43 deacetylation was observed after extreme overexpression of the mainly cytoplasmic

269 NAD+ dependent deacetylase SIRT2 ${ }^{33}$, so we cannot rule out overexpression artefacts. However,

270 moderate levels of the nuclear deacetylase SIRT1 ${ }^{33}$ were sufficient to counteract TDP-43 acetylation at

271 K136. It is of note that TDP-43 was reported to bind to the 3'-UTR of SIRT1 mRNA and regulate SIRT1

272 expression ${ }^{35}$. Thus, the nuclear deacetylase SIRT1 may be engaged in a regulatory network with TDP-

273 43. To see if the reduction of acetylation correlated with a reduction in the amount of aggregates of

274 [acK136]TDP-43, we cotransfected those sirtuins with an impact in TDP-43 acetylation (SIRT1 and SIRT2)

275 as well as other sirtuins with similar level of expression (SIRT3 and SIRT6). When compared to cells

276 without any sirtuin overexpression, SIRT1 and 2 caused a significant reduction in the number of cells with

277 inclusions, even though some level of TDP-43 acetylation was detected (Fig. 7b, c). 


\section{Discussion}

In ALS only $4 \%$ of familial cases have mutations on TDP-43 and they are even rarer in FTLD $(<1 \%)^{36,37}$. PTMs of wtTDP-43 might promote disease but it is still unknown if they are a cause or consequence of

282 TDP-43 aggregation 4,18 . Here we show that TDP-43 nuclear import and RNA binding are dynamic 283 processes that can be regulated by distinct PTMs. Specifically, we have discovered that acetylation of the core NLS residue K84 partially reduces nuclear import of TDP-43, leading to the accumulation of cytosolically mislocalized protein, which is considered as an early step of TDP-43 pathogenesis. Moreover, we identify $\mathrm{K} 136$ in the RRM1 as a potential regulatory site for TDP-43 RNA binding and splicing activity. Although we could confirm the stippled distribution and reduced CFTR splicing activity for the previously reported [K145Q]TDP-4318,19, the effects for K136 located directly within an essential RRM1 site appeared stronger in our experimental system. Acetylation can modulate the binding to RNA of other hnRNPs and therefore likely for TDP-43 as well ${ }^{38,39}$. Such loss of functional RNA binding may lead to LLPS and eventually the formation of intranuclear TDP-43 aggregates. Acetylation of lysine residues in

292 the RRM1 could trigger aggregation of TDP-43, driving it into phase-separated droplets that eventually coalesce into bigger aggregates. This was also very recently proposed by $\mathrm{Yu}$ et al. who looked at [K145Q/K192Q]TDP-43, two acetylation sites that interfere with TDP-43 RNA-processing functions ${ }^{40}$. The phase-separated [K145Q/K192Q]TDP-43 can become less fluid and presumably turn into solid aggregates. Our findings support this progression for [acK136]TDP-43 and identify SIRT1 as a deacetylase counteracting this process. When combined with $\Delta$ NLS, [acK136]TDP-43 also showed a tendency to form cytosolic aggregates, which are much more commonly found in human patients than intranuclear inclusions ${ }^{41}$. Thus, acetylation of K136 might disengage TDP-43 from functional hnRNP complexes, liberating the protein to unmix into liquid phases. Such LLPS transitions may lead to TDP-43 self-aggregation and the formation of pathological inclusions. The observation that SIRT1 can deacetylate [acK136]TDP-43 indicates that this is a dynamic process that can be regulated in cells. The balance of TDP-43 protein lysine acetyltransferases and deacetylases might determine its physiological functions and when derailed, could lead to LLPS and pathological TDP-43 aggregation in disease. Modulation of this pathway could offer novel therapeutic approaches for the treatment of FTLD and ALS. 
307 Residue K136 was recently discovered as a potential TDP-43 sumoylation site ${ }^{17}$. Consistent with this 308 study by Maurel et al. (2020) using TDP-43 GFP fusion proteins, we found that $6 x$ His-tagged 309 [K136R]TDP-43 formed intranuclear inclusions. The similarities in behaviour between K136R and K136Q 310 TDP-43 mutants were puzzling, suggesting that instead of mimicking the presence or lack of acetylation, 311 the mutants were introducing backbone changes in TDP-43. Point mutagenesis is a broadly used 312 approach to study the cellular effects of protein lysine acetylation but in some cases can fail to rescue 313 protein function ${ }^{42}$. In addition, glutamine and arginine contain delocalized electrons that are critical for the 314 formation of phase-separated entities ${ }^{43}$. This provides an explanation for the stronger occurrence of 315 phosphorylated aggregates in K136R/Q TDP-43. Amber suppression has its own disadvantages in terms 316 of efficiency and availability of synthetic amino acids, in addition to the possibility of side effects. 317 Transcriptomic analysis of stably amber suppressed cells does not show upregulation of ER stress 318 markers ${ }^{44}$. Another benefit of this approach is the possibility of removing the modification in vivo via deacetylases (see Fig. 7).

321 Unfortunately, we could not detect phosphorylation and ubiquitination of the amber suppressed 322 [acK136]TDP-43 (not shown), in contrast to the K136Q mutant TDP-43. We think there are three factors 323 that can influence this result. First, compared with transfected mutant [K136Q]TDP-43, expression of 324 amber suppressed TDP-43 is much lower (see Fig. 6a), potentially below detection limit of other PTMs. 325 The lower concentration might also not be sufficient to seed protein aggregation, phosphorylation and 326 ubiquitination. Second, K136-acetyl in the amber suppressed TDP-43 can be removed by endogenous 327 deacetylases such as SIRT1. This would attenuate any downstream effects of this acetylation. And third, 328 as shown by Wang et al. glutamines favour the hardening of phase-separated RNA-binding molecules ${ }^{22}$. 329 While these key differences could be behind the lack of the strongest markers of TDP-43, amber 330 suppression still could help identifying K136 as a residue crucial for RNA splicing and LLPS in TDP-43. 
332 In our MS analysis we did not detect the previously reported acetylations at K145 and K192 in the RNA-

333 binding domain of TDP- $43^{18}$. Although our experimental setups were similar, perhaps the use of the

334 protein aggregation optimized QBI-293 subclone in their studies made the difference, whereas our study

335 in HEK293E cells revealed acetylation of K121 and K136 in RRM1. Moreover, the MS detection of K145

336 acetylation was done with $\Delta$ NLS mutant TDP-43 ${ }^{18,19}$ lacking the K84 acetylation site we detected with

337 wtTDP-43. It is possible that differential lysine acetylation events occur in cytosolic and nuclear TDP-43

338 pools. Antibodies directed against [acK145]TDP-43 stained neuropathological inclusions in ALS but not

339 FTLD-TDP18. Yet, MS analysis of TDP-43 PTMs in 2 ALS cases failed to confirm all of these lysine

340 acetylations but instead found K82 acetylation in one ALS case ${ }^{45}$. Unfortunately, the acK136 peptide is

341 poorly immunogenic, and we obtained only one single hybridoma clone that produced antibody with

342 sufficient selectivity and sensitivity for our cell culture experiments, but not for human disease samples

343 (not shown). Thus, the human disease relevance for lysine acetylated TDP-43 remains to be proven.

$345 \quad$ Materials and Methods

\section{$346 \quad$ Antibodies}

347 In this study the following antibodies were used for Western blot (WB) and immunofluorescence staining

348 (IF): rat anti-[acK84]TDP-43 (WB, 1:10 supernatant; IF,1:2; this work), rat anti-[acK136]TDP-43 (WB, 1:10

349 supernatant; IF,1:2; this work; mouse anti-6xHis (WB, 1:10000; IF, 1:1000; Amersham 27-4710-01), rabbit 350 pan-acetylated lysine (WB, 1:1000; Cell Signalling \#9441), rabbit mAb mix pan-acetylated lysine (WB,

351 1:1000; Ac-K-100, Cell Signalling \#9841), mouse anti-Flag HRP-coupled (WB, 1:10000; Sigma \#A8592),

352 mouse anti-GAPDH (WB, 1:50000; clone 6C5, Biodesign International \#H86504M), rabbit anti-Hsp90

353 (WB, 1:1000; Cell Signalling \#4874), rat anti-[pS409/410]TDP-43 (WB, 1:10; IF, 1:50; clone 1D3), rabbit

354 anti-TDP-43 (WB, 1:8000; IF, 1:1000; ProteinTech 10782-2-AP), mouse anti-TDP-43 (WB, 1:2000; IF,

355 1:1000; Abnova \#H00023435), mouse anti-tubulin (WB, 1:10000; Sigma \#T-5168), mouse anti-ubiquitin

356 (WB, 1:4000; Millipore \#MAB1510), mouse anti-YY1 (WB, 1:2000; clone H-10, Santa Cruz Biotechnology

357 \#7341), mouse anti- $\beta$-actin (WB, 1:50000; clone AC-15, Sigma \#A5441); rabbit anti-mouse HRP-coupled 
(WB, 1:10000, Amersham \#NIF825); goat anti-rabbit HRP-coupled (WB, 1:10000, Amersham \#NIF824); donkey anti-rat HRP-coupled (WB, 1:10000, Jackson Immunoresearch, 712-035-150) and secondary Alexa Fluor 488-, 568-, 647- conjugated antibodies for IF produced in goat were from Invitrogen (1:1000).

\section{cDNA constructs}

wtTDP-43 was cloned into pCMV 5'6His (Clontech) via Sall/Notl. Acetyl-mimic glutamine and acetyl-dead arginine substitutions were introduced by site-directed mutagenesis and cloned into pCMV 5'6His (Sall/Notl). The cloning and mutagenesis primers are listed in supplementary table 3 . The mutations were introduced via a 2-step PCR mutagenesis, where TDP-43 gene was amplified in two fragments: from the start of the gene to the mutant codon, and from the mutant codon to the end. These two fragments were used as a template for a third PCR reaction in this case amplifying the whole length of TDP-43 and introducing restriction sites at both ends.

For the EGFP tagged constructs, pEGFP c1 wtTDP-43 was used as a template ${ }^{46}$. K136R and K136Q mutations were introduced via site-directed mutagenesis and cloned into pEGFP c1 via BamHI and HindIII.

375 For the C-terminally tagged amber suppression constructs, TDP-43 was cloned from the pCMV 5' 6His 376 construct previously mentioned into a pCMV 3'6His vector via Sall and Bsd120I. The amber stop codons 377 from constructs K84TAG and K136TAG TDP-43 were introduced via 2-step point mutagenesis. For the N378 terminally tagged amber suppression constructs, TDP-43 was cloned from the amber suppression C379 terminally tagged constructs previously mentioned into the vector E400 via Nhel and BamHI. The 380 plasmids E400_pAS1_4x7SKPyIT_EF1_In_IRES_Bsd (E400) (https://benchling.com/s/seq-

382 (https://benchling.com/s/seq-6fpczrM3tMNE9fZOP2nk). Constructs lacking the C-terminal part and with mutated NLS were in addition to K136TAG were cloned from previously described plasmids 6 and cloned into pCMV 3'6His vector via Sall and Bsd120l. Cloning primers are listed in supplementary Table 2. 
386 Plasmids containing sirtuins were ordered from Addgene, and all of them were produced by Eric Verdin.

387 The plasmids ordered were SIRT1 Flag (Addgene plasmid \# 13812; RRID:Addgene_13812), SIRT2 Flag

388 (Addgene plasmid \# 13813; RRID:Addgene_13813), SIRT3 Flag (Addgene plasmid \# 13814;

389 RRID:Addgene_13814), SIRT4 Flag (Addgene plasmid \# 13815; RRID:Addgene_13815), SIRT5 Flag

390 (Addgene plasmid \# 13816; RRID:Addgene_13816), SIRT6 Flag (Addgene plasmid \# 13817;

391 RRID:Addgene_13817), and SIRT7 Flag (Addgene plasmid \# 13818; RRID:Addgene_13818). All sirtuin

392 plasmids were characterised by North et al. ${ }^{47}$.

393

394 Plasmids containing HDACs 2-5, 7 and 8 were ordered from Addgene. The plasmids ordered were 395 HDAC2 Flag (Addgene plasmid \# 36829 ; http://n2t.net/addgene:36829 ; RRID:Addgene_36829), HDAC3

396 Flag (Addgene plasmid \# 13819 ; http://n2t.net/addgene:13819 ; RRID:Addgene_13819), HDAC4 Flag

397 (Addgene plasmid \# 13821 ; http://n2t.net/addgene:13821 ; RRID:Addgene_13821), HDAC5 Flag

398 (Addgene plasmid \# 13822 ; http://n2t.net/addgene:13822 ; RRID:Addgene_13822), and HDAC7 399 (Addgene plasmid \# 13824 ; http://n2t.net/addgene:13824 ; RRID:Addgene_13824). HDAC2 plasmid was 400 characterised by Reyon et al. ${ }^{48}$, HDAC3 plasmid was characterized by Emiliani et al. ${ }^{49}$ and HDAC4, 5, 7 401 and 8 were characterized by Fischle et al. ${ }^{50}$. HDAC1 and HDAC6 plasmids were generated by Fiesel et $402 \quad$ al. $^{29}$

403

\section{Cell culture and transfections}

HEK293E were cultured in Dulbecco's Modified Eagle Medium (DMEM) containing 10\% Fetal Bovine 406 Serum (FBS), at $37^{\circ} \mathrm{C}$ in $5 \%$ CO2. Stably TDP-43 Knocked-down cells were generated and characterized 407 by previously ${ }^{25}$. Cells were plated at different concentrations corresponding to the surface of the plate for 408 different experiments. Transfection of plasmids was done 24 hours after plating using FuGENE6 409 (Promega) at a DNA/FuGENE ratio of 1:4.5 according to manufacturer's instructions.

\section{Amber suppression}

412 HEK293E cells were plated and cultured in DMEM with 10\% FBS and transfected with FuGENE6 413 (Promega) 24 hours later. 24 or 48 hours after transfection the media was substituted with DMEM with 
414 10\%FBS and 5mM Ne-Acetyl-L-lysine (A4021, Sigma). Cells were lysed or fixed after being 24 hours in

415 the presence of acetylated lysine. When mentioned, Ex 527 (CAS 49843-98-3, Santa Cruz Biotechnology)

416 was dissolved in DMSO and added to the cultured cells 24 hours before lysis to a final concentration of 1

417 or $10 \mu \mathrm{M}$.

\section{Cell lysis, solubility fractionation, and Western blotting}

420 Cells were collected with a cell scraper and lysed in urea lysis buffer (10mM Tris pH 8, 100mM NaH2PO4, $4218 \mathrm{M}$ Urea). For the sequential extraction cells were first lysed with RIPA buffer $(50 \mathrm{mM} \mathrm{Tris} / \mathrm{HCl} \mathrm{pH} 8$, $422150 \mathrm{mM} \mathrm{NaCl}, 1 \% \mathrm{NP}-40,0.5 \%$ deoxycholate, $0.1 \%$ SDS, $10 \mathrm{mM} \mathrm{NaPPi}$ ) with proteinase inhibitor cocktail 423 (Roche). Samples were centrifuged at $18000 \mathrm{rpm}$ for 15 minutes, leaving the RIPA-soluble proteins in the 424 supernatant. The remaining pellet was then lysed in urea buffer, and insoluble material was removed with 425 a centrifugation step at $18000 \mathrm{rpm}$ for 15 minutes. DNA was sheared with a 23-gauge needle. Protein 426 concentration in lysates in urea buffer was quantified with a Bradford Protein assay kit (Biorad) and 427 lysates in RIPA buffer with BCA protein assay kit (Pierce). 3xLaemmli loading buffer with 100mM DTT was 428 added to the samples and afterwards they were boiled at $95^{\circ}$ for 5 minutes. The denatured samples were 429 then subjected to Western blot analysis. Denatured samples were loaded and run in polyacrylamide gels. 430 The gels were then blotted using the wet-transfer Trans-Blot@ Cell system from Bio-Rad. Proteins were 431 transferred to Hybond-P polyvinylidene difluoride membranes (Millipore). Membranes were blocked with $4325 \%$ non-fat milk/TBS-T for an hour at room temperature (RT). Primary antibody incubation took place 433 overnight at $4^{\circ} \mathrm{C}$. The membranes were washed and incubated with a HRP-coupled secondary antibody 434 for 1-2 hours at RT. Membranes were washed and proteins were detected with Immobilon Western 435 chemiluminescent HRP substrate (Millipore) using the ChemiDoc XRS+ Imaging System.

438 Rats were immunized with two ovalbumin-coupled synthetic peptides at the same time: one with amino 439 acids corresponding to residues 79-89 of TDP-43, with K84 acetylated (C-KDNKR(Ac)KMDETD); and the 440 second one corresponding to residues 131-141, with K136 acetylated (C-LMVQV(Ac)KKDLKT). Animals 441 were sacrificed and splenocytes were fused with mouse myeloma cells. Hybridoma supernatants were 442 tested for binding to acetylated and non-acetylated peptides by ELISA. Those supernatants that were 
443 positive for the acetylated peptides and negative for the non-acetylated peptides were further validated on

444 lysates of cells expressing either wild-type or amber suppressed TDP-43 by Western blot analysis (dilution

$4451: 10$ ) and immunofluorescence staining (dilution 1:2). Hybridoma clones TDACA 9G9 (IgG2c/k) against

446 [acK84]TDP-43, TDACB 23B3 (IgG1/k) (used only in supplementary Fig. 4) and TDACB 14D4 (IgG2b/k)

447 against [acK136]TDP-43 were stably established by limiting dilution cloning.

\section{Immunofluorescence staining}

450 Cells were plated in 6-well plates and grown for 24 hours. They were then transfected and 6 hours after 451 transfection they were transferred (diluted 1:10) to coverslips in a 24-well plate coated with poly-D-lysine 452 (PDL) and collagen. Cells then grew for 24-72 hours before fixation with 4\% PFA/PBS for 20 minutes at 453 RT depending on the experiment. Permeabilization of the cell membrane was done with $1 \%$ Triton-X454 100/PBS for 5 minutes at RT. Blocking was done with 10\% Normal Goat Serum in PBS, for 1 hour at RT.

455 The primary antibody incubations were done at the already mentioned concentrations, in 1\%BSA/PBS for 4562 hours at RT. The secondary antibody incubation was done in 1\% BSA/PBS for 1 hour. Mounting was 457 done using $40 \mu \mathrm{l}$ of Dako mounting media per slide, following a $4^{\circ}$ incubation overnight. 458 Immunofluorescence images were acquired using a Zeiss fluorescence microscope with Apotome 459 attachment (Axio imager z1 stand).

\section{Fluorescence recovery after photobleaching} HEK293E cells were plated on glass bottom chambered sides (Lab Tek, 154526) coated with PDL and collagen and transfected with C-terminally EGFP-tagged wt or K136Q TDP-43 after 24 hours. Imaging and 464 photobleaching was done with a Zeiss LSM510 META confocal microscope, using a x63/1.4 oil objective.

465 ROls were bleached for 1 s using a $405 \mathrm{~nm}$ and a $488 \mathrm{~nm}$ laser at maximum power output. Cells were 466 continuously imaged for 3 minutes after bleaching. Reference ROIs were measured from unbleached cells 467 to correct for photobleaching due to fluorescence imaging. 
470 HEK293E cells were plated on glass bottom chambered slides coated with PDL. They were transfected

471 with C-terminally EGFP-tagged wt or K136Q TDP-43 after 24 hours. While imaging cells were kept in a

472 temperature controlled chamber at $37^{\circ}$ with $5 \% \mathrm{CO}_{2}$. Imaging was done with an Axio observer $\mathrm{Z1}$.

473

\section{LC-MS/MS}

475 Mass spectrometry data were obtained from the analysis of the data generated by Hans et al. ${ }^{17}$. In short,

476 6xHis tagged TDP-43 was purified via nickel beads pulldown and further purified by size in a SDS-PAGE

477 gel, visualised with Coomasie Brilliant Blue staining. Trypsin (Promega) digestion was performed in-gel.

478 Extracted peptides were analyzed by LC-MS/MS using a nanoflow HPLC system (Ultimate 3000 RSLC;

479 ThermoFisher Scientific) coupled to an Orbitrap Fusion (ThermoFisher Scientific) tandem mass

480 spectrometer. Peptides were separated by reversed C-18 chromatography and 120-min gradients. MS1

481 spectra were acquired in the Orbitrap at 120,000 resolution. Precursors were selected with the top speed

482 method and 3-s cycle time. HCD-MS2 spectra were acquired with linear ion trap detection. For database

483 search, tandem mass spectra were extracted by MSConvert (ProteoWizard version 3.0.6938). Charge

484 state deconvolution and de-isotoping were not performed. All MS/MS samples were analyzed using

485 Mascot (Matrix Science; version 2.5.1). Mascot was set up to search the SwissProt database (selected for

486 Homo sapiens, 2015_03, 20203 entries) assuming the digestion enzyme trypsin.

\section{RNA-protein pulldown}

489 High performance liquid-chromatography-purified RNA oligonucleotides 5'-(UG)12-3' and 5'-(UC)12-3' 490 were ordered from Sigma. The oligonucleotides were biotinylated using the Pierce RNA 3' End 491 Desthiobiotinylation kit (Cat $n^{\circ}$ 20163) with small variations. For each $1 \mathrm{nmol}$ of biotinylated cytidine 492 bisphosphate 100pmol of RNA were added to the biotinylation reaction. Biotinylated RNA oligos were 493 purified using chloroform:isoamyl. For the RNA-protein pulldown the Pierce Magnetic RNA-Protein 494 Pulldown kit (Cat $n^{\circ}$ 20164) was used with small variations. Cells were lysed in a NP-40 buffer (50mM $495 \mathrm{NaH} 2 \mathrm{PO} 4 \mathrm{pH} 8.0 ; 300 \mathrm{mM} \mathrm{NaCl} ; 1 \% \mathrm{NP}-40$ ) and $400 \mu \mathrm{g}$ of protein was used for each RNA-protein binding reaction. After 1 -hour incubation proteins were eluted in $25 \mu \mathrm{l}$ Laemmli buffer. 
HEK293E sh ${ }^{\text {TDP-43 }}$ cells were plated in $10 \mathrm{~cm}$ plates. After 24 hours, cells were transfected with the respective plasmids. After 48 hours, cells were lysed in Native NiNTA lysis buffer $\left(50 \mathrm{mM} \mathrm{NaH}_{2} \mathrm{PO}_{4}\right.$, $300 \mathrm{mM} \mathrm{NaCl}, \mathrm{pH} 8,10 \mathrm{mM}$ imidazole). $500-800 \mu \mathrm{g}$ of protein were incubated with NiNTA beads at $4^{\circ} \mathrm{C}$ overnight. Beads were washed with Native NiNTA lysis buffer with $20 \mathrm{mM}$ imidazole. After washing, proteins were eluted in Binding buffer (10mM HEPES, $20 \mathrm{mM} \mathrm{KCl}, 1 \mathrm{mM} \mathrm{MgCl} 2,1 \mathrm{mM}$ DTT, $5 \%$ glycerol) containing $300 \mathrm{mM}$ imidazole. The purified protein was incubated at various concentrations with $2 \mathrm{nM}$ of biotinylated poly-(UG) 12 RNA for 30 minutes at room temperature. The resulting complex was run through a Bio-Dot@ SF Microfiltration apparatus (Bio Rad, Cat $n^{\circ}$ : 170-6542) loaded with a nitrocellulose and a positively charged nylon membrane. Membranes were then cross-linked at $120 \mathrm{~J} / \mathrm{m}^{2}$ and then labelled RNA was detected using the Chemiluminescent Nucleic Acid Detection module kit (Thermofisher, cat $\mathrm{n}^{\circ}$ : 89880) according to the manufacturer's instructions.

\section{Nickel pulldown of $6 x H i s-t a g g e d ~ T D P-43$}

512 Cells were plated in $10 \mathrm{~cm}$ plates for 24 hours before transfection. 72 hours after transfection cells were

513 lysed in urea lysis buffer containing $10 \mathrm{mM}$ imidazole. DNA was sheared with a 23-gauge needle. After 514 quantifying the amount of protein in the lysates with a Bradford Protein assay kit (Biorad), $500-800 \mu g$ of 515 protein were incubated with Ni-NTA agarose beads (Qiagen) at $4^{\circ} \mathrm{C}$ overnight. Beads were washed with 516 Urea wash buffer $(10 \mathrm{mM}$ Tris $\mathrm{pH} 6.3,100 \mathrm{mM}$ NaH2PO4, 8M Urea) containing 20mM imidazole. 517 Afterwards proteins were eluted in $3 \times$ Laemmli buffer at $95^{\circ}$ for 10 minutes. Eluted proteins were analysed 518 in Western blot.

\section{RNA extraction and RT-PCR}

521 sh TDP-43 TDP-43 HEK293E cells were plated in 6-well plates and after 24 hours were double-transfected 522 with the corresponding TDP-43 construct and a CFTR minigene construct ${ }^{12}$ in a proportion of 2:1. After 72 523 hours RNA was isolated using the RNeasy Mini Kit (Qiagen) following the indications from the provider.

524 From the isolated RNA cDNA was obtained using the Transcriptor High Fidelity cDNA Synthesis Kit 525 (Sigma) following the protocol of the provider. The cDNA of interest was then amplified via a standard 526 PCR and the product was run in a $2 \%$ agarose gel. DNA was visualised using Midori Green from Biozym 527 and the ChemiDoc XRS+ Imaging System. 


\section{Statistical analyses and molecular representations}

530 Cellprofiler 4.1.3 was used for quantification of immunofluorescence pictures. Statistical significance was 531 calculated using Microsoft Excel 2016. P values below 0.01 were considered significant. Unpaired t-tests 532 were performed to compare two variables and assess significance. Chi-squared test was used to assess 533 significance in the immunofluorescence experiments. $n$ values detailed in each figure legend. Graphical 534 representations of TDP-43 mutants and acetylated forms was made with Pymol (version 2.4.0). Post 535 translational modifications were modulated using the PyTMs plugin by Warnecke et al. ${ }^{51}$

\section{Acknowledgements}

538 We would like to thank Dr. Ivana Nikić-Spiegel and Dirk Schwarzer for helpful discussions, Dr. Petra Frick 539 for the validation of the acetyl-TDP-43 antibodies, Dr. Sven Geisler for excellent technical advice, Stefan 540 Hauser for help with Live Cell Imaging, and Anna Lechado Terradas for critical comments on the 541 manuscript. This work was supported by the NOMIS Foundation; German Research Foundation (DFG) 542 grant KA1675/3-2; the German Center for Neurodegenerative Diseases (DZNE) within the Helmholtz 543 Association; and the Hertie Foundation.

\section{Author contributions}

546 JGM designed and performed most experiments and analyzed the results. FH assisted the study and 547 provided TDP-43 reagents. FvZ and CJG performed MS and analyzed the data. RF generated the 548 antibodies against acetylated TDP-43. SE provided the amber suppression methodology and reagents.

549 AS established the FRAP assay. EB provided the CFTR reporter minigene and methodological support for 550 splicing and RNA binding assays. MN contributed to the study design and provided neuropathological 551 tools. MN and PJK acquired funding. PJK conceptualized and supervised the study. JGM and PJK wrote 552 the draft and all co-authors edited the paper. 


\section{Conflict of interest}

555 The authors declare that they have no conflict of interest with the contents of this article.

\section{References}

\begin{tabular}{|c|c|c|}
\hline 558 & 1 & $\begin{array}{l}\text { Buratti, E. \& Baralle, F.E. The multiple roles of TDP-43 in pre-mRNA processing and gene } \\
\text { expression regulation (Taylor and Francis Inc., 2010), Vol. 7, pp. } 420-429 .\end{array}$ \\
\hline 560 & 2 & Sephton, C.F., Cenik, B., Cenik, B.K., Herz, J., \& Yu, G., TDP-43 in central nervous system \\
\hline 561 & & development and function: clues to TDP-43-associated neurodegeneration. Biol Chem 393 (7), \\
\hline 62 & & 589-594 (2012). \\
\hline 563 & 3 & Ratti, A. \& Buratti, E., Physiological functions and pathobiology of TDP-43 and FUS/TLS proteins. J \\
\hline 564 & & Neurochem 138 Suppl 1 (S1), 95-111 (2016). \\
\hline 565 & 4 & $\begin{array}{l}\text { Neumann, M. et al., Ubiquitinated TDP-43 in frontotemporal lobar degeneration and } \\
\text { amyotrophic lateral sclerosis. Science } 314(5796), 130-133(2006) \text {. }\end{array}$ \\
\hline $\begin{array}{l}567 \\
568 \\
569\end{array}$ & 5 & $\begin{array}{l}\text { Arai, T. et al., TDP-43 is a component of ubiquitin-positive tau-negative inclusions in } \\
\text { frontotemporal lobar degeneration and amyotrophic lateral sclerosis. Biochemical and }\end{array}$ \\
\hline 69 & & Biophysical Research Communications 351 (3), 602-611 (2006). \\
\hline 70 & 6 & Winton, M.J. et al., Disturbance of nuclear and cytoplasmic TAR DNA-binding protein (TDP-43) \\
\hline 71 & & induces disease-like redistribution, sequestration, and aggregate formation. J Biol Chem 283 (19), \\
\hline 572 & & $13302-13309$ (2008). \\
\hline $\begin{array}{l}573 \\
574\end{array}$ & 7 & $\begin{array}{l}\text { Pinarbasi, E.S. et al., Active nuclear import and passive nuclear export are the primary } \\
\text { determinants of TDP-43 localization. Sci Rep } 8(1), 7083(2018) \text {. }\end{array}$ \\
\hline 575 & 8 & Ayala, Y.M. et al., Structural determinants of the cellular localization and shuttling of TDP-43. $J$ \\
\hline 576 & & Cell Sci 121 (Pt 22), 3778-3785 (2008). \\
\hline 577 & 9 & Tollervey, J.R. et al., Characterizing the RNA targets and position-dependent splicing regulation \\
\hline 578 & & by TDP-43. Nat Neurosci 14 (4), 452-458 (2011). \\
\hline 579 & 10 & Buratti, E. \& Baralle, F.E., Characterization and functional implications of the RNA binding \\
\hline 580 & & properties of nuclear factor TDP-43, a novel splicing regulator of CFTR exon 9. J Biol Chem 276 \\
\hline 581 & & (39), 36337-36343 (2001). \\
\hline 582 & 11 & Buratti, E. et al., Nuclear factor TDP-43 and SR proteins promote in vitro and in vivo CFTR exon 9 \\
\hline 583 & & skipping. EMBO J 20 (7), 1774-1784 (2001). \\
\hline 584 & 12 & Prasad, A., Sivalingam, V., Bharathi, V., Girdhar, A., \& Patel, B.K., The amyloidogenicity of a C- \\
\hline 585 & & terminal region of TDP-43 implicated in Amyotrophic Lateral Sclerosis can be affected by anions, \\
\hline 586 & & acetylation and homodimerization. Biochimie 150, 76-87 (2018). \\
\hline 587 & 13 & Gao, F.B., Almeida, S., \& Lopez-Gonzalez, R., Dysregulated molecular pathways in amyotrophic \\
\hline 588 & & lateral sclerosis-frontotemporal dementia spectrum disorder. EMBO J 36 (20), 2931-2950 (2017). \\
\hline $\begin{array}{l}589 \\
590\end{array}$ & 14 & $\begin{array}{l}\text { Hasegawa, M. et al., Phosphorylated TDP- } 43 \text { in frontotemporal lobar degeneration and } \\
\text { amyotrophic lateral sclerosis. Ann Neurol } 64 \text { (1), 60-70 (2008). }\end{array}$ \\
\hline 591 & 15 & Neumann, M. et al., Phosphorylation of S409/410 of TDP-43 is a consistent feature in all s \\
\hline 592 & & d familial forms of TDP-43 proteinopathies. Acta Neuropathol 117 (2), 137-149 (20) \\
\hline
\end{tabular}


Hans, F., Eckert, M., von Zweydorf, F., Gloeckner, C.J., \& Kahle, P.J., Identification and characterization of ubiquitinylation sites in TAR DNA-binding protein of $43 \mathrm{kDa}$ (TDP-43). J Biol Chem 293 (41), 16083-16099 (2018).

17 Maurel, C. et al., A role for SUMOylation in the Formation and Cellular Localization of TDP-43 Aggregates in Amyotrophic Lateral Sclerosis. Mol Neurobiol 57 (3), 1361-1373 (2020). Nat Commun 6, 5845 (2015). pathology is suppressed by an HSF1-dependent chaperone program. Nat Commun 8 (1), 82 (2017).

Maharana, S. et al., RNA buffers the phase separation behavior of prion-like RNA binding proteins. Science 360 (6391), 918-921 (2018). Mann, J.R. et al., RNA Binding Antagonizes Neurotoxic Phase Transitions of TDP-43. Neuron 102 (2), 321-338 e328 (2019).

Wang, J. et al., A Molecular Grammar Governing the Driving Forces for Phase Separation of Prion-like RNA Binding Proteins. Cell 174 (3), 688-699 e616 (2018).

Bentmann, E. et al., Requirements for stress granule recruitment of fused in sarcoma (FUS) and TAR DNA-binding protein of 43 kDa (TDP-43). J Biol Chem 287 (27), 23079-23094 (2012).

Zhang, Y.J. et al., The dual functions of the extreme N-terminus of TDP-43 in regulating its biological activity and inclusion formation. Hum Mol Genet 22 (15), 3112-3122 (2013).

Voigt, A. et al., TDP-43-mediated neuron loss in vivo requires RNA-binding activity. PLoS One 5 (8), e12247 (2010).

Lukavsky, P.J. et al., Molecular basis of UG-rich RNA recognition by the human splicing factor TDP-43. Nat Struct Mol Biol 20 (12), 1443-1449 (2013).

Chiang, C.H. et al., Structural analysis of disease-related TDP-43 D169G mutation: linking enhanced stability and caspase cleavage efficiency to protein accumulation. Sci Rep 6, 21581 (2016).

Neumann, M., Molecular neuropathology of TDP-43 proteinopathies. Int J Mol Sci 10 (1), 232246 (2009).

Fiesel, F.C. et al., Knockdown of transactive response DNA-binding protein (TDP-43) downregulates histone deacetylase 6. EMBO J 29 (1), 209-221 (2010).

Bryson, D.I. et al., Continuous directed evolution of aminoacyl-tRNA synthetases. Nat Chem Biol 13 (12), 1253-1260 (2017).

Kawakami, I., Arai, T., \& Hasegawa, M., The basis of clinicopathological heterogeneity in TDP-43 proteinopathy. Acta Neuropathologica 138 (5), 751-770 (2019).

Conicella, A.E., Zerze, G.H., Mittal, J., \& Fawzi, N.L., ALS Mutations Disrupt Phase Separation Mediated by $\alpha$-Helical Structure in the TDP-43 Low-Complexity C-Terminal Domain. Structure 24 (9), 1537-1549 (2016).

Seto, E. \& Yoshida, M., Erasers of histone acetylation: the histone deacetylase enzymes. Cold Spring Harb Perspect Biol 6 (4), a018713 (2014).

Tang, B.L. Could Sirtuin Activities Modify ALS Onset and Progression? (Springer New York LLC, 2017), Vol. 37, pp. 1147-1160.

$\mathrm{Yu}, \mathrm{Z}$. et al., Neurodegeneration-associated TDP-43 interacts with fragile $\mathrm{X}$ mental retardation protein (FMRP)/Staufen (STAU1) and regulates SIRT1 expression in neuronal cells. J Biol Chem 287 (27), 22560-22572 (2012).

Renton, A.E., Chio, A., \& Traynor, B.J., State of play in amyotrophic lateral sclerosis genetics. Nat Neurosci 17 (1), 17-23 (2014). 
64037 Baizabal-Carvallo, J.F. \& Jankovic, J. Parkinsonism, movement disorders and genetics in frontotemporal dementia (Nature Publishing Group, 2016), Vol. 12, pp. 175-185. Gal, J. et al., The Acetylation of Lysine-376 of G3BP1 Regulates RNA Binding and Stress Granule Dynamics. Mol Cell Biol 39 (22) (2019). Zhang, W., Sun, Y., Liu, W., Dong, J., \& Chen, J., SIRT1 mediates the role of RNA-binding protein QKI 5 in the synthesis of triglycerides in non-alcoholic fatty liver disease mice via the PPARalpha/FoxO1 signaling pathway. Int J Mol Med 43 (3), 1271-1280 (2019). $\mathrm{Yu}, \mathrm{H}$. et al., HSP70 chaperones RNA-free TDP-43 into anisotropic intranuclear liquid spherical shells. Science 371 (6529) (2021). Geser, F., Lee, V.M., \& Trojanowski, J.Q., Amyotrophic lateral sclerosis and frontotemporal lobar degeneration: a spectrum of TDP-43 proteinopathies. Neuropathology 30 (2), 103-112 (2010). Choudhary, C. et al., Lysine acetylation targets protein complexes and co-regulates major cellular functions. Science 325 (5942), 834-840 (2009). for Biochemistry and Molecular Biology Inc., 2019), Vol. 294, pp. 7115-7127. Elsasser, S.J., Ernst, R.J., Walker, O.S., \& Chin, J.W., Genetic code expansion in stable cell lines enables encoded chromatin modification. Nat Methods 13 (2), 158-164 (2016). sclerosis brains. Sci Rep 6 (1), 23281 (2016).

671 


\section{Figure legends}

675 Figure 1. TDP-43 is acetylated and its acetylation at K84 may affect its nuclear-cytoplasmic trafficking while acetylation at K136 causes a nuclear droplet-like distribution.

677 a) HEK293E cells were transfected with $6 x$ His tagged TDP-43, which was purified with Ni-NTA beads and 678 subjected to MS. The residues marked in the domain structure of TDP-43 were found to be acetylated 679 (black arrows). K145 has been reported to be acetylated in the literature but we could not find it in our 680 study (grey arrow). b) Immunostaining of HEK293E cells transfected with 6xHis tagged wtTDP-43, acetyldead (K84R, K136R and K145R) or acetyl-mimics (K84Q, K136Q and K145Q). Scale bar represents 10 $682 \mu \mathrm{m} . \mathrm{N}=3$. c) Classification of $6 \times \mathrm{H}$ is tagged TDP -43 transfected cells from $\mathbf{b}){ }^{* * *}=p<0.001$ as measured by Chi squared test. The classification was made based on the subcellular compartment with predominantly more TDP-43. 300 transfected cells per group classified. d) Cytoplasmic and nuclear fractions from HEK293E cells transfected with 6xHis tagged wt, K84R, K84Q or Flag tagged $\Delta$ NLS TDP. 43. These fractions were analyzed by Western blot. $\mathrm{N}=2$. Hsp90 and $\mathrm{YY} 1$ were used as cytoplasmic and nuclear markers respectively. e) Quantification of the proportion of TDP-43 in the cytoplasm normalized to wtTDP-43. $N=2 .{ }^{*}=p<0.05,{ }^{*}=p<0.01$. pathological phosphorylation.

a) ShTDP-43-HEK293E cells were transfected with 6xHis tagged TDP-43 (wt, K136R and K136Q) or Flagtagged FFLL TDP-43. RIPA soluble and insoluble fractions were separated and analyzed by Western blotting. $\mathrm{N}=3$. b) ShTDP-43-HEK293E cells were transfected with $6 \mathrm{xHis}$ tagged TDP-43 (wt, K136R and K136Q). Proteasome activity was inhibited by treatment with $20 \mu \mathrm{M}$ MG-132 for 6 h, which caused wtTDP43 ubiquitination (lane 3). 6xHis tagged TDP-43 was purified with Ni-NTA beads and analyzed on Western

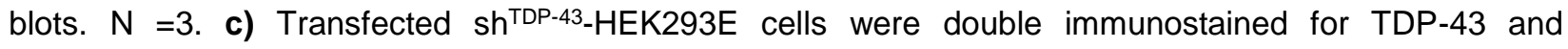
phosphorylated TDP-43 (S409/410). Scale bar represents 10 $\mu \mathrm{m}$. 
a) ShTDP-43-HEK293E cells were transfected with an empty plasmid, wt or K136Q TDP-43. The lysates were then incubated with biotinylated (UC)12 or (UG)12 RNA oligomeres. Protein-RNA mix was incubated with magnetic streptavidin beads. Bound proteins were eluted and analyzed by Western blotting. b) Increasing concentrations of $6 x$ His purified protein was incubated with biotinylated (UG) 12 oligomers. The resulting complex was analyzed by a filter binding assay. Membranes were incubated with HRP-coupled streptavidin. c) Quantification of 3 replicates of filter-binding assay of wt and K136Q TDP-43 to biotinylated (UG) ${ }_{12}$ RNA oligomers. ${ }^{* *}=p<0.01$. d) Western blot of the protein levels before and after the

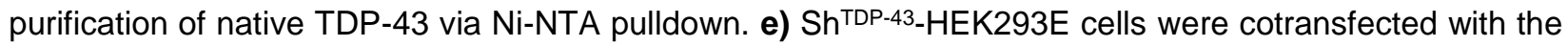
specified TDP-43 constructs and a plasmid containing the CFTR minigene. RNA was extracted and splicing of CFTR exon 9 was assessed via rtPCR (upper panel). Protein levels are shown in the lower panel. $N=3 . \mathbf{f})$ Quantification of 3 replicates looking at the effect of mutations at different lysines of TDP43 on the splicing of CFTR exon 9. ${ }^{* *}=p<0.01$.

Figure 4. Amber suppression-mediated acetylation at K136 of TDP-43 prevents CFTR splicing, nuclear import.

a) HEK293E cells cotransfected with a plasmid containing acKRS and 4xtRNATAG (E451) and with TDP-43 with an amber stop codon at different positions and a C-terminal $6 \mathrm{xHis}$ tag. Media was changed to $5 \mathrm{mM}$ acK-containing media 24 hours before lysis. Protein lysates were analysed via Western blot. b) Cells were cotransfected with the E451 plasmid and different C-terminally 6xHis tagged TDP-43 constructs containing amber suppression codons. Cells were fixed after $24 \mathrm{~h}$ in the presence of $5 \mathrm{mM}$ acK. Scale bar represents

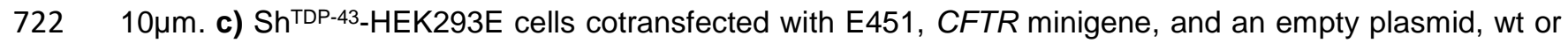
723 K136TAG TDP-43. After 24 hours in the presence of $5 \mathrm{mM}$ acK, RNA was extracted and the splicing of

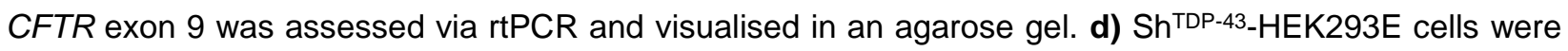
cotransfected with E451 plasmid and different C-terminally tagged TDP-43 constructs. Lanes 3-5 have an 
deletion of the C-terminus. Cells were treated with $5 \mathrm{mM}$ acK for $24 \mathrm{~h}$ before lysis. e) ShTDP-43-HEK293E

728 cells were cotransfected with E451 and different C-terminally tagged TDP-43 constructs. Cells were fixed

729 and immunolabeled after $24 \mathrm{~h}$ in the presence of $5 \mathrm{mM}$ acK. Scale bar represents $10 \mu \mathrm{m}$. f) Classification of

730250 cells per group cotransfected with E451 plasmid and different 6xHis C-terminally tagged TDP-43

731 constructs. The classification was made based on the subcellular compartment with predominantly more

732 TDP-43.

Figure 5. [K136Q]TDP-43 droplets have reduced mobility, growing in size and fusing over time.

a) Representative images of FRAP analysis of HEK293 cells transfected with C-terminally EGFP-tagged wt and K136Q TDP-43. Two different groups of [K136Q]TDP-43-EGFP droplets based on size were determined. Time in seconds. Scale Bar represents $5 \mu \mathrm{m}$. b) Quantification of FRAP images. 3 cells mutants. 3 cells quantified per group. ${ }^{*}=p<0.05$. d) Selected frames from green fluorescence live cell imaging of cells expressing [K136Q]TDP-43-EGFP. Close-up of granules fusing (white arrows). Time in seconds. Scale bar represents $5 \mu \mathrm{m}$ e) Histogram of K136Q TDP-43 aggregate size in HEK293E fixed 24, 48 or 72 hours after transfection. 100 transfected cells analyzed for each time point. ${ }^{* *}=p<0.01$.

Figure 6. Antibodies against acetylated TDP-43 specifically recognize the modified forms.

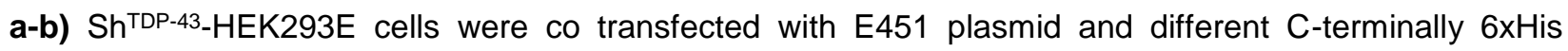
tagged TDP-43 constructs. After $24 \mathrm{~h}$ in the presence of $5 \mathrm{mM}$ of acK cells were lysed. Samples were Western probed with antibodies against acetylated K84 (clone 9G9) and K136 (clone 14D4), respectively. tagged TDP-43 constructs. After $24 \mathrm{~h}$ in the presence of $5 \mathrm{mM}$ of acK cells were fixed and immunostained for $6 \mathrm{xHis}$ or acetylated TDP-43. Scale bar represents $10 \mu \mathrm{m}$.

Figure 7. Sirtuins 1 and 2 can deacetylate K136 of TDP-43 and prevent its aggregation. 
753 a) ShTDP-43-HEK293E cells were cotransfected with E451, K136TAG TDP-43 and SIRT1 or SIRT2. 24h

754 before lysis, $5 \mathrm{mM}$ AcK and DMSO, $1 \mu \mathrm{M}$ or $10 \mu \mathrm{M}$ Ex527 containing media was supplied to the cells. b)

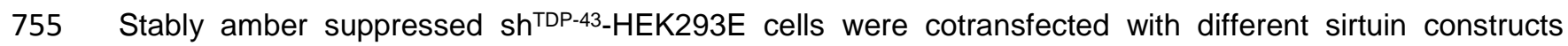

756 together with K136TAG TDP-43. Cells were fixed after 24h in the presence of $5 \mathrm{mM}$ acK. c) Quantification of

757 the number of cells with TDP-43 positive inclusions. 200 cells recorded per condition.

Supplementary Table 1. TDP-43 peptides found by MS to be acetylated. Longer chain lysine modifications like propionylation and butyrylation were not detected.

Supplementary Table 2. List of mutagenesis and cloning primers.

Supplementary Figure 1. Immunostaining of HEK293E cells previously transfected with 6xHis tagged non acetyl-mimics (K79R and K121R) or acetyl-mimics (K79Q and K121Q). Scale bar represents $10 \mu \mathrm{m}$. $\mathrm{N}=3$.

Supplementary Figure 2. a) Structure of TDP-43 RRM1 in contact with RNA (PDB: 4BS2) with circles represent points of contact between TDP-43 amino acids. Red circles represent clashes between residue 136 and other objects. subcellular distribution.

a) Western blot of HEK293E cells transfected with E451 containing a Flag-tagged acKRS, RNATAG and 
777 acK in the media. b) Double immunostaining of HEK293E cells transfected with Flag-tagged acKRS,

778 RNATAG and different C-terminal 6xHis-tagged TDP-43 constructs. Cells were fixed after 24h in the 779 presence of $5 \mathrm{mM}$ acK in the media. Scale bar represents $10 \mu \mathrm{m}$.

\section{Supplementary Figure 4. HDAC and Sirtuin effect on K136 acetylation of TDP-43.}

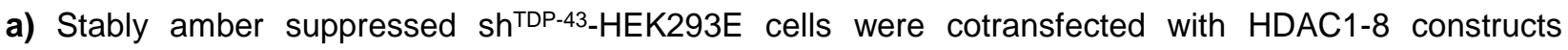
together with K136TAG TDP-43. Cells were lysed after $24 \mathrm{~h}$ in the presence of $5 \mathrm{mM}$ acK. Protein levels were assessed via Western blot. HDAC1 and 6 were myc-tagged, while HDAC2, 3, 4, 5,7 and 8 were or HDAC6 constructs together with K136TAG TDP-43 or wtTDP-43. Cells were lysed after $24 \mathrm{~h}$ in the presence of $5 \mathrm{mM}$ acK. Protein levels were assessed via Western blot. $\mathrm{N}=2$ c) Stably amber suppressed

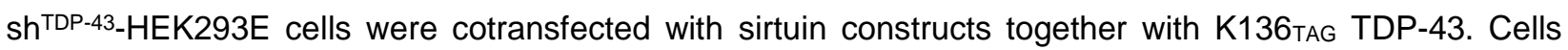
were lysed after $24 \mathrm{~h}$ in the presence of $5 \mathrm{mM}$ acK. Protein levels were assessed via Western blot. $\mathrm{N}=3$.

Supplementary video 1 and 2. Live imaging of HEK293E cells 48h after transfection with [K136Q]TDP43-EGFP. Cells were kept at $37^{\circ} \mathrm{C}$ and $5 \% \mathrm{CO}_{2}$ during the experiment. Imaging took place every $5 \mathrm{~min}$ for 4h.

Supplementary video 3. Live imaging of HEK293E cells 48h after transfection with [K136Q]TDP-43EGFP. Cells were kept at $37^{\circ} \mathrm{C}$ and $5 \% \mathrm{CO}_{2}$ during the experiment. Imaging took place every $2 \mathrm{~min}$ for $1 \mathrm{~h}$. 

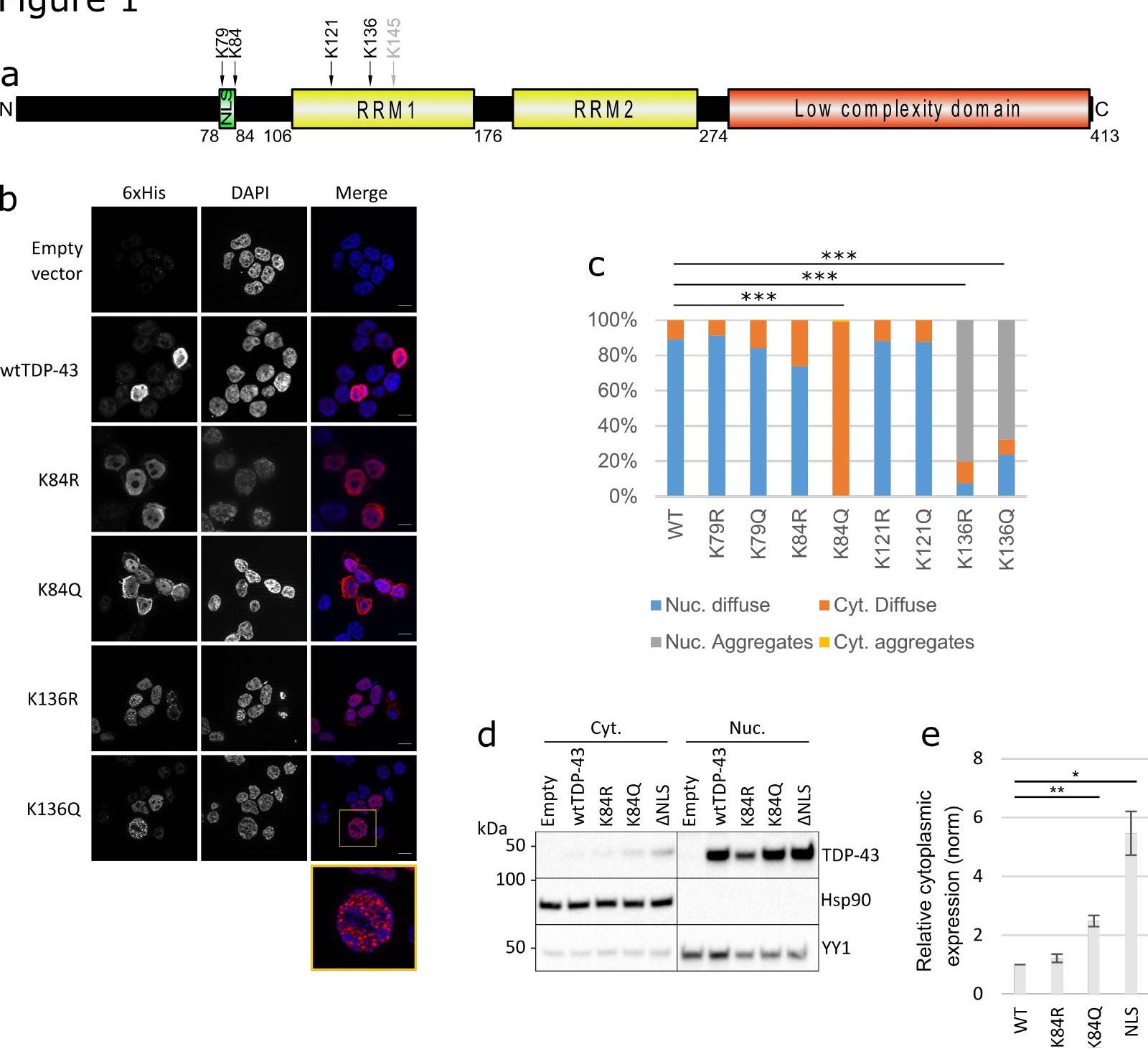
Figure 2

bioRxiv preprint doi: https://doi.org/10.1101/2020.05.26.104356; this version posted May 27, 2021. The copyright holder for this preprint (which was not certified by peer review) is the author/funder, who has granted bioRxiv a license to display the preprint in perpetuity. It is made
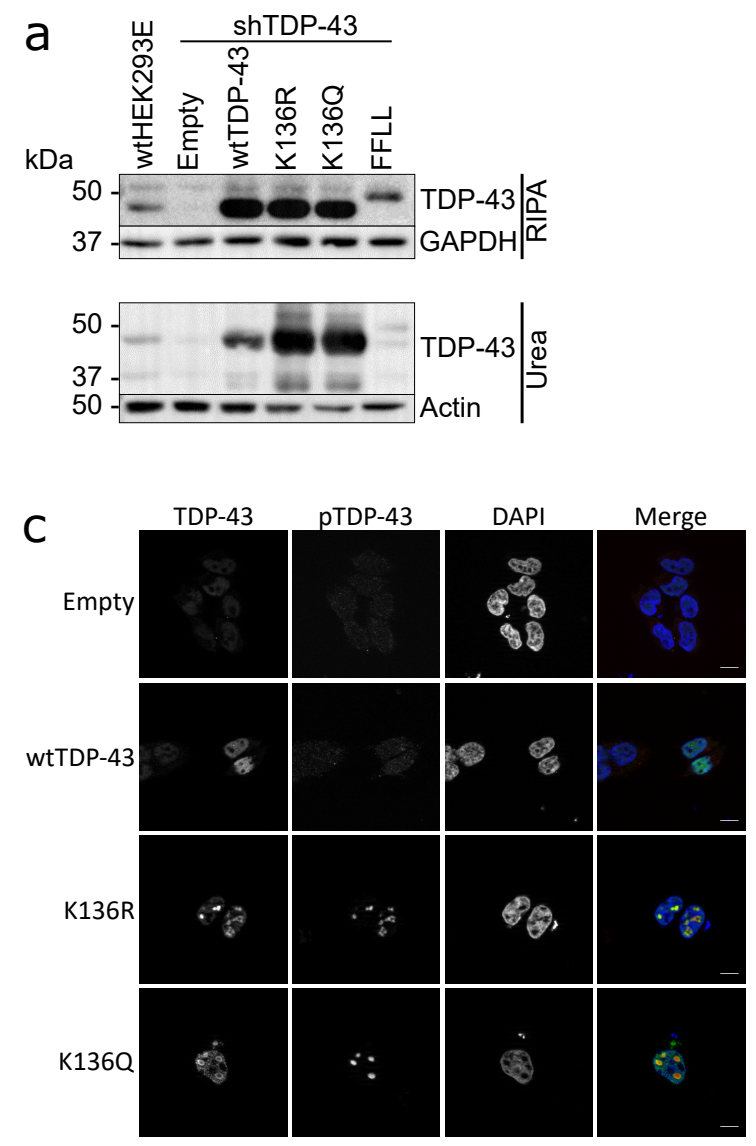
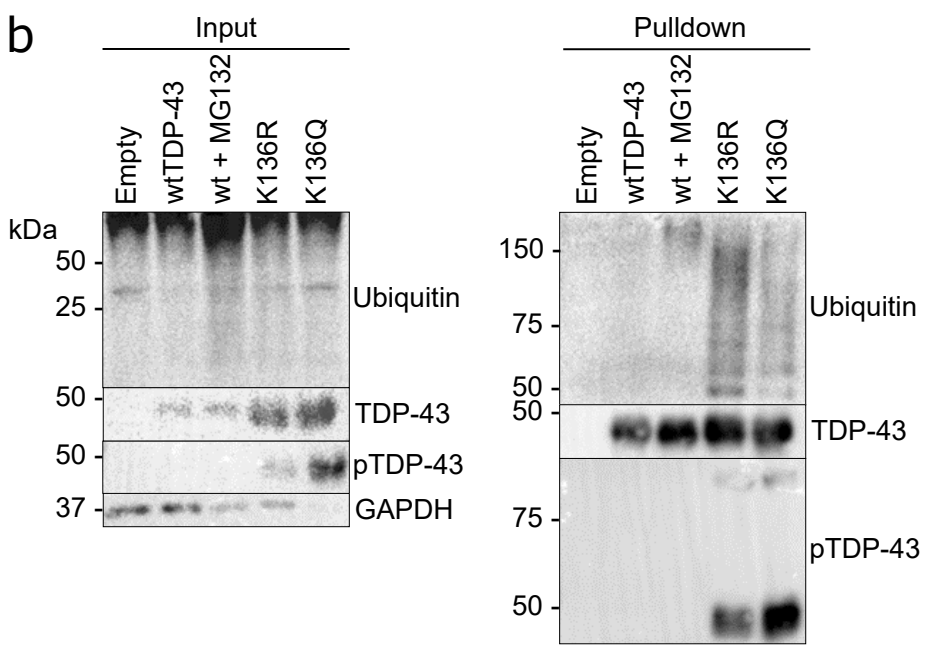


\section{Figure 4}

a

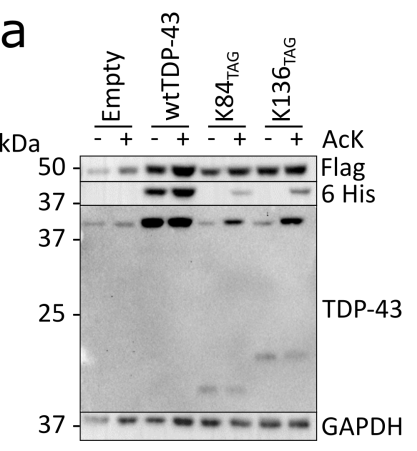

C

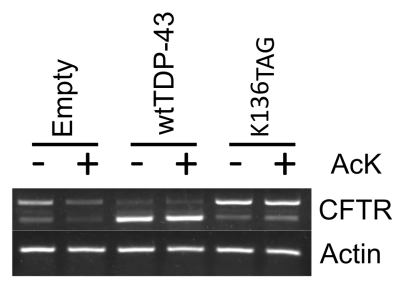

d 1: Empty 2: wtTDP43 3:ANLS 4: K84TAG 5: $\triangle \mathrm{C}$-ter

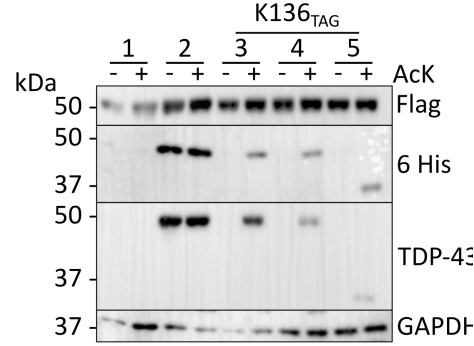

b

Empty

-Ack

Empty

+ AcK

wtTDP-43

-AcK
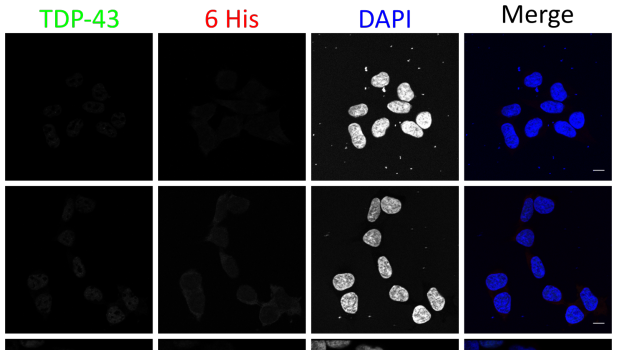

wtTDP-43

+ AcK
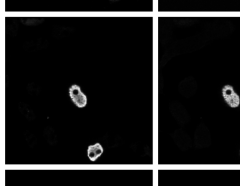

옹

$\mathrm{K} 84_{\mathrm{TAG}} \mathrm{O}$

+ AcK

$\mathrm{K} 136_{\mathrm{TA}}$

-AcK

$\mathrm{K} 136_{\mathrm{TAG}}$

+ AcK
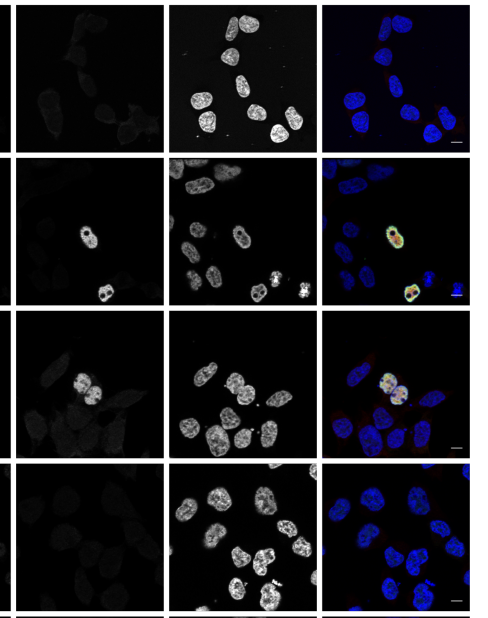

$\therefore 0$

$10 \%$

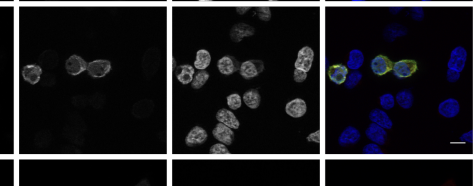

-

\%

6

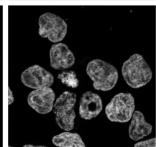

e

TDP-43

6 His

DAPI

Merge

$\mathrm{K} 136_{\mathrm{TAG}}$

K136

$\Delta$ Cter
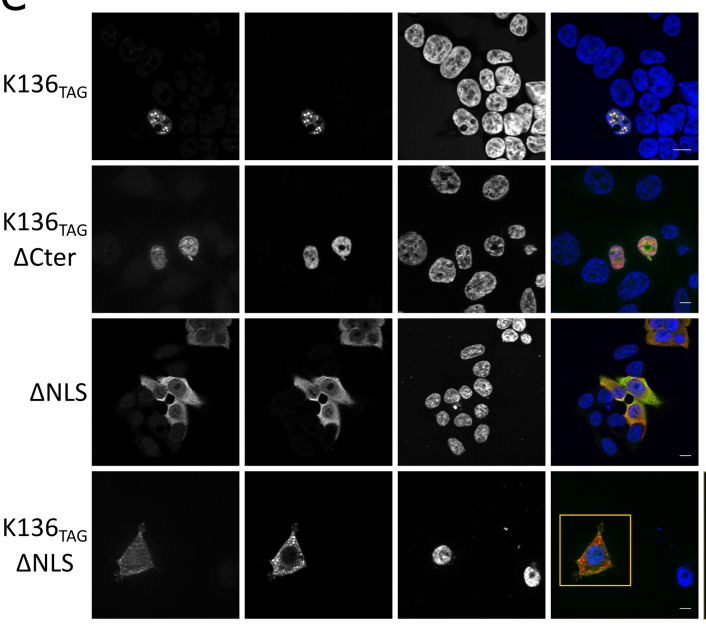

f
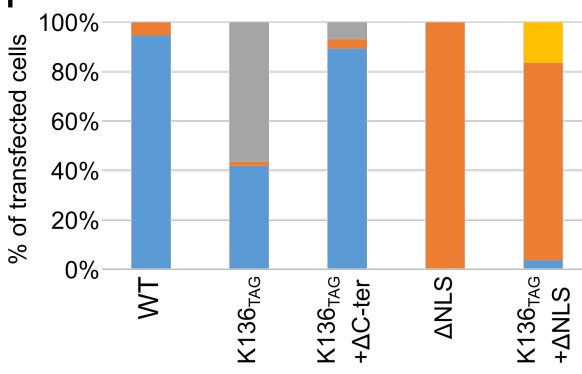

Nuc. diffuse $=$ Cyt. diffuse Nuc. agg. Cyt. agg. 


\section{Figure 5}

a

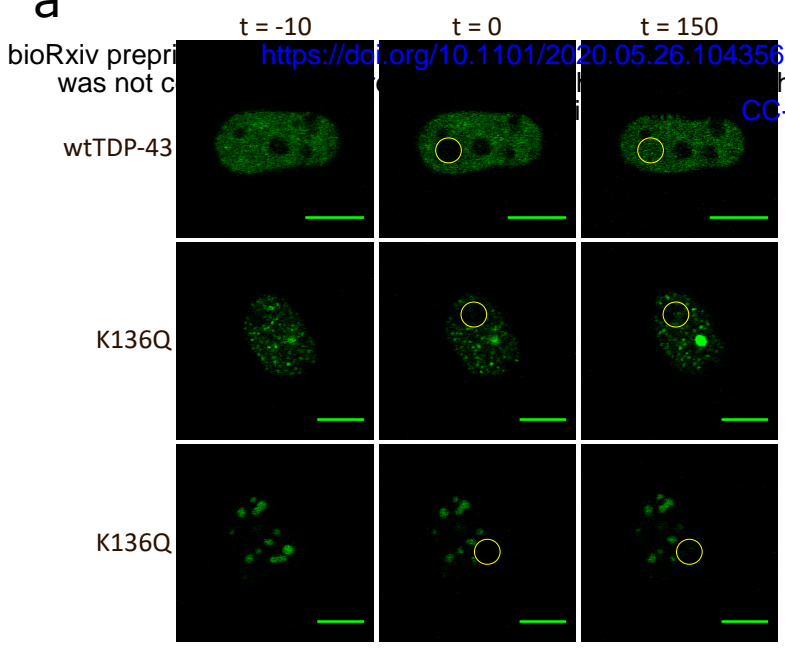

b

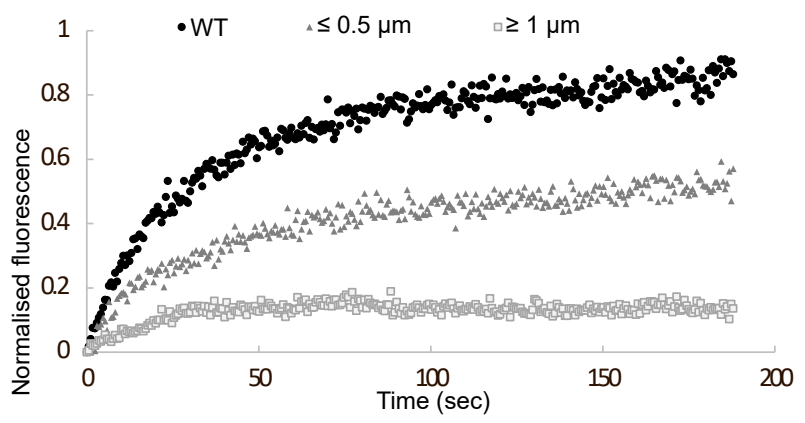

C

this version posted May 27, 2621 . The eopyright holder for this preprint (which has granted bioRxiv a license to display the preprint in perpetuity. It is made -BY-NC-ND 4.0 International license.

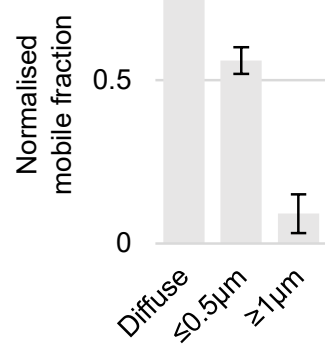

d

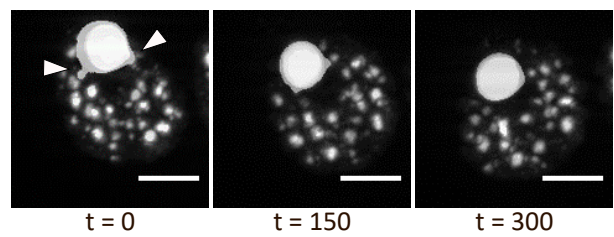

e

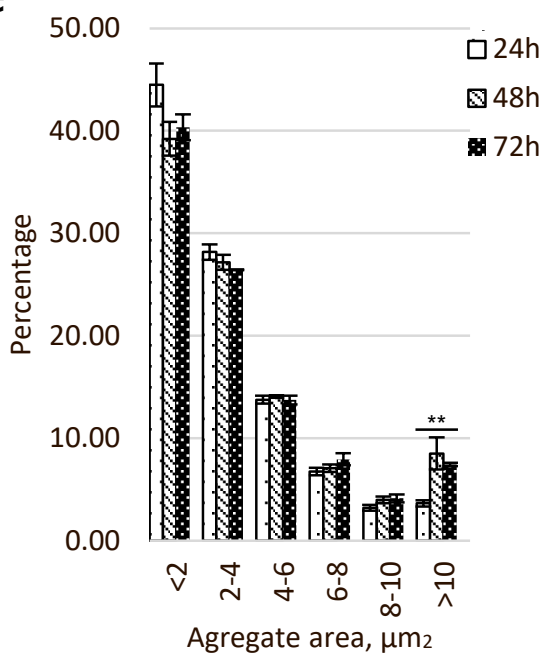


Figure 6

bioRxiv preprint doi: https://doi.org/10.1101/2020.05.26.104356; this version posted May 27, 2021. The copyright holder for this preprint (which wasnot certified by peer review) is the author/fyder, who has granted bioRxiv a license to display the preprint in perpetuity. It is made
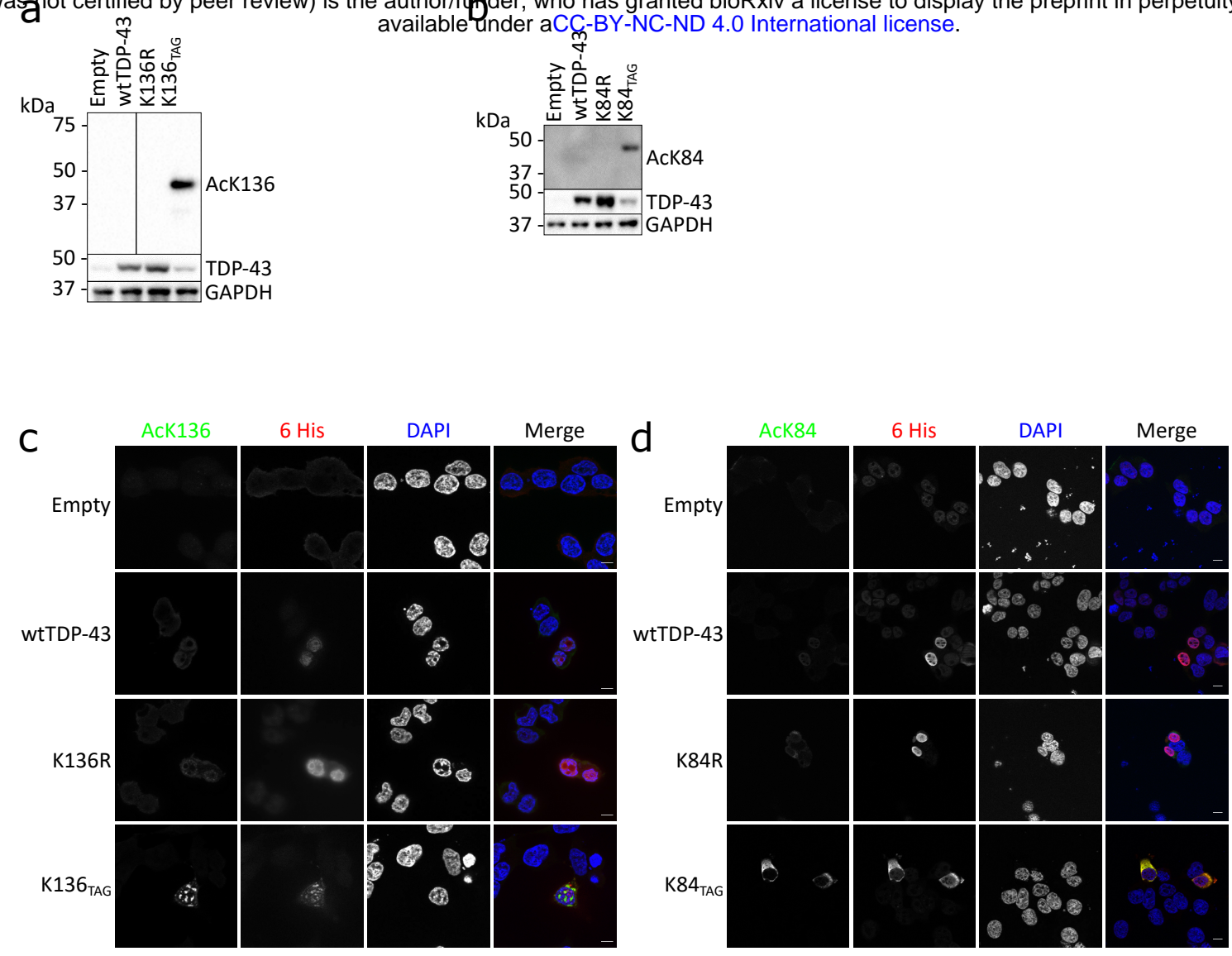
Figure 7
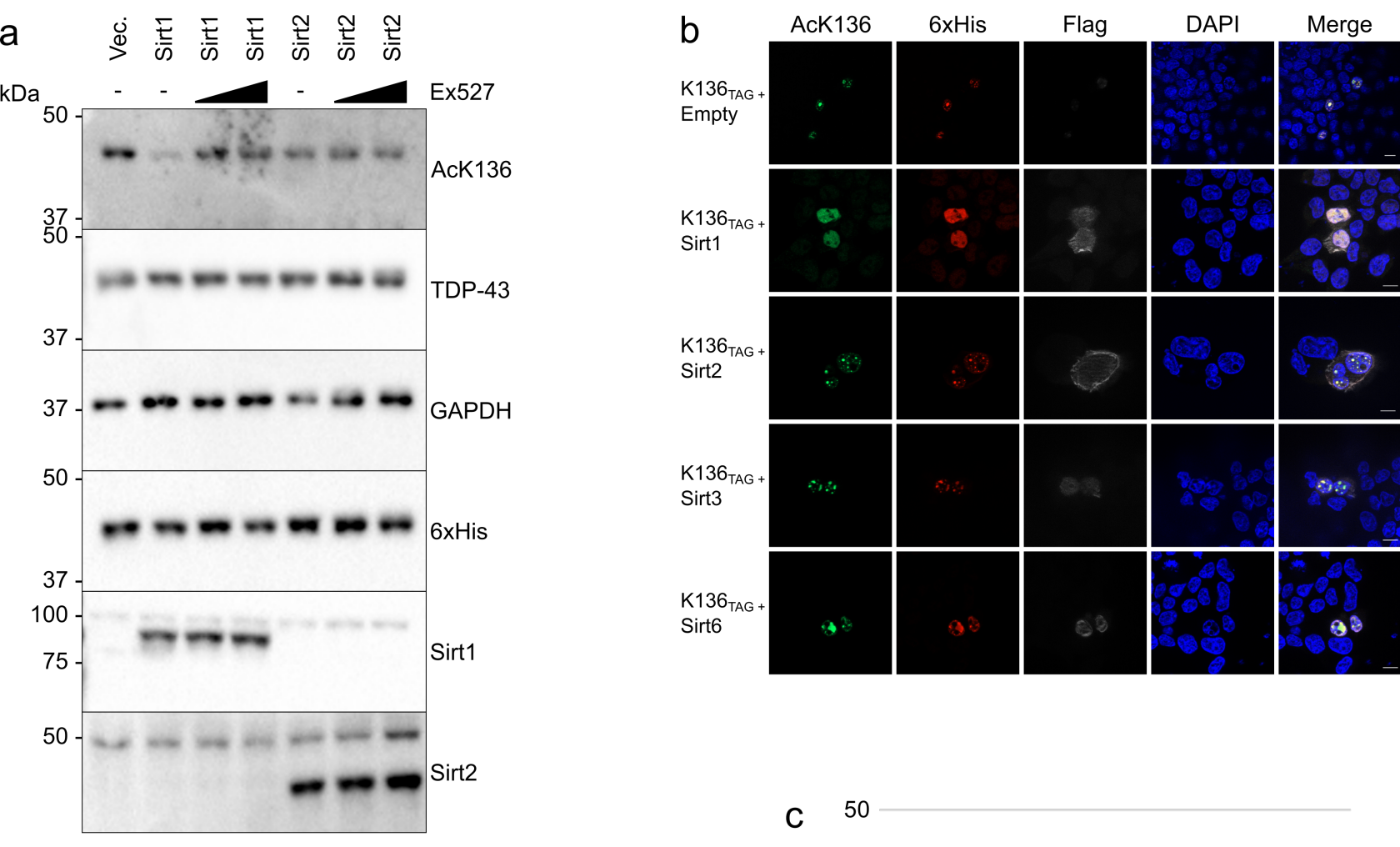

C 50

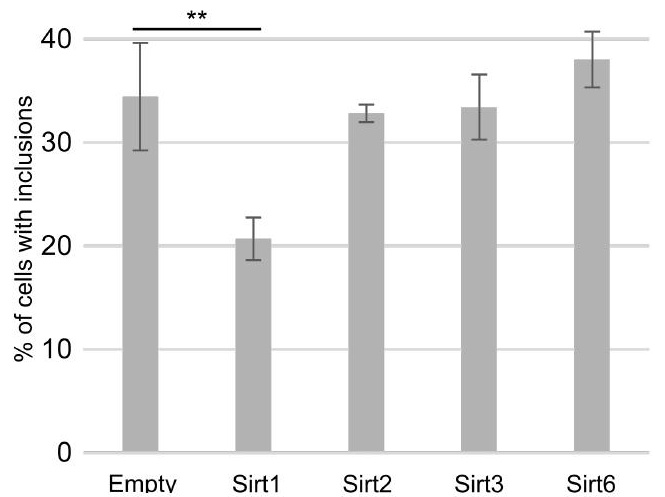

